\title{
Respostas fisiológicas de estresse no matrinxã (Brycon amazonicus) após exercício físico intenso durante a captura
}

\author{
Márcio Aquio HOSHIBA¹, Flávio Daolio GONÇALVES², Elisabeth Criscuolo URBINATI³ \\ RESUMO \\ Para determinar as respostas de estresse do matrinxã após perseguição com puçá, juvenis $(26,7 \pm 6,7 \mathrm{~g})$ foram aclimatados em \\ caixas plásticas e submetidos aos tratamentos: Controle (sem perseguição), Perseguição por 2 minutos, Perseguição por 5 \\ minutos, Perseguição por 10 minutos (quatro repetições, $\mathrm{N}=8$ /tratamento). Amostras de sangue foram coletadas 15, 30 e 60 \\ minutos após a perseguição para determinação do cortisol, glicose, sódio, cloreto, potássio, hematócrito, hemoglobina, número \\ total de eritrócitos e osmolaridade. O perfil das respostas após o exercício físico dos peixes não mostrou as alterações típicas \\ do estresse. Até 60 minutos após o estímulo, não ocorreram alterações nos níveis sanguíneos de cortisol, glicose e potássio \\ nos peixes dos diferentes tratamentos. Os níveis de cloreto foram reduzidos 15 minutos após a natação forçada, enquanto os \\ níveis do sódio mais baixos foram registrados 60 minutos depois. Houve redução da osmolaridade a partir dos 30 minutos \\ após o estímulo, independente do tempo de perseguição. A natação forçada não interferiu nos indicadores hematológicos, \\ corroborando os outros indicadores usados. Dessa forma, o exercício intenso dos peixes por até 10 minutos não foi estímulo \\ suficiente para gerar respostas de estresse, sugerindo que o matrinxã é bastante resistente ao manejo de criação.
}

PALAVRAS-CHAVE: Natação forçada, Estresse, Matrinxã, Captura, Cortisol

\section{Physiological stress responses of matrinxã (Brycon amazonicus) after chasing}

\section{ABSTRACT}

This study reports the stress responses of matrinxã after chasing. Juvenile fish $(26.7 \pm 6.7 \mathrm{~g})$ were adapted to $100 \mathrm{~L}$ plastic boxes and submitted to the treatments: Control (no chasing), Chasing for $2 \mathrm{~min}$, Chasing for $5 \mathrm{~min}$, Chasing for $10 \mathrm{~min}$ (four boxes/ treatment, 32 fish). Blood was drawn 15, 30 and $60 \mathrm{~min}$ after chasing to determine levels of cortisol, glucose, sodium, chloride, potassium, calcium, hematocrit, hemoglobin, erythrocytes number and osmolality. Matrinxã responses after forced swimming did not show the typical stress responses. There were no changes in blood cortisol, glucose, potassium and calcium levels in fish from any treatment until $60 \mathrm{~min}$ after chasing. Blood chloride levels were lower $15 \mathrm{~min}$ after chasing while the lowest levels of sodium were registered $60 \mathrm{~min}$ after the forced swimming. Osmolality dropped $30 \mathrm{~min}$ after chasing, regardless the period of chasing. The intense swimming did not affect hematological indicators confirming the other physiological indicators. The results suggest that the intense swimming provoked by chasing up to $10 \mathrm{~min}$ was not intense enough to promote stress responses in matrinxã indicating that the species is very resistant to the farming handling.

KEY WORDS: Forced swimming, Stress, Matrinxã, Capture, Cortisol

\footnotetext{
1 Universidade Federal do Amazonas. e-mail: tokudazoo@gmail.com

2 e-mail: fdaolio@yahoo.com.br.

${ }^{3}$ Professor adjunto da FCAV/UNESP. e-mail: bethurb@caunesp.unesp.br
} 


\section{INTRODUÇÃO}

A prática da criação expõe os peixes a condições de manejo que alteram seu equilíbrio fisiológico, tais como a captura (Grutter \& Pankhurst, 2000), o transporte (Robertson et al., 1987; Urbinati et al., 2004), o confinamento (Rocha et al., 2004) e as mudanças ambientais (Pickering \& Pottinger, 1987). Essas exposiçōes, que podem ocorrer de forma aguda ou crônica, são constantes no processo produtivo, gerando respostas fisiológicas características do estresse. Os peixes respondem a uma variedade de estressores com o fenômeno chamado síndrome da adaptação geral descrito inicialmente em mamíferos (Selye, 1950). As reações fisiológicas dos peixes são usualmente divididas em primárias e secundárias (Mazeaud et al., 1977) e são utilizadas como indicadores de estresse (Morgan \& Iwama, 1997). O aumento do cortisol no sangue é uma importante resposta primária, enquanto a elevação da glicemia e alteraçôes hematológicas, iônicas e da osmolaridade são efeitos secundários característicos (McDonald \& Milligan, 1997; Morgan \& Iwama, 1997; Wendelaar Bonga, 1997).

A captura dos peixes, uma das etapas mais agressivas do manejo da criação, se caracteriza pelo caráter agudo e severo (Clearwater \& Pankhurst, 1997). A acumulação dos estressores que ocorre durante a captura pode causar estresse aumentado no peixe. Tanto o exercício físico da perseguição (Milligan et al., 2000), como as injúrias causadas pelo contato físico com a rede ou outros animais (Ross \& Ross, 1999), além da hipóxia provocada pela exposição aérea do peixe (Arends et al., 1999), são estressores isolados, embora promovam respostas de estresse semelhantes (Selye, 1950). A quantificação da resposta gerada por cada componente da captura permite estabelecer estratégias de manejo para reduzir os estímulos mais estressantes do processo. Tanto a captura com puçás em condições de laboratório, quanto com redes em viveiros de criação, provoca fuga dos peixes e natação forçada e extenuante.

Estudos sobre estresse em peixes brasileiros de interesse para a criação vêm sendo desenvolvidos (Gomes et al., 2003a, b; Urbinati \& Carneiro, 2004). O matrinxã (Brycon amazonicus), espécie da Bacia Amazônica que está sendo criada em muitas regiões do país, tem sido estudado como modelo biológico de respostas a estressores, incluindo-se transporte (Carneiro \& Urbinati, 2001,a,b; Urbinati \& Carneiro, 2001; Carneiro \& Urbinati, 2002; Urbinati et al., 2004); densidade de estocagem (Rocha et al., 2004) e respostas comportamentais (Ide et al., 2003). Este trabalho avaliou o efeito isolado da perseguição de matrinxã, por meio de respostas hormonais, metabólicas, eletrolíticas e hematológicas, sobre a homeostasia do matrinxã durante a captura.

\section{MATERIAL E MÉTODOS}

\section{ANIMAIS E TRATAMENTOS}

Os peixes utilizados para o experimento eram procedentes do mesmo lote e foram doados pelo Centro de Aquicultura do Caunesp. O experimento foi realizado no Laboratório de Peixes do Departamento de Morfologia e Fisiologia Animal, sendo utilizados 320 peixes $(26,7 \pm 6,7 \mathrm{~g})$, aclimatados em 40 caixas plásticas de $100 \mathrm{~L}$ (8 peixes por caixa), com renovação contínua de água e ar. Os mesmos foram submetidos aos seguintes tratamentos: Controle (sem perseguição com puçá), Perseguição por 2 minutos, Perseguição por 5 minutos, Perseguição por 10 minutos. Cada tratamento foi aplicado a peixes de quatro caixas (total de 32 peixes). Os peixes foram amostrados 15, 30 e 60 minutos depois da perseguição. Foram amostrados os 32 peixes de cada tratamento, no entanto para a realização das analises foi realizado o pool de cada $2(n=16)$, devido a pouca quantidade de material de cada amostragem. O experimento foi realizado em 2 etapas (dias diferentes), pois o número de repetiçôes foi muito grande, inviabilizando a amostragem num único dia. Em cada dia, foram amostrados 2 repetiçôes de todos os tratamento e em todos os tempos de amostragem pós-perseguição.

Durante o experimento, registrou-se, em todas as caixas, a temperatura, o nível de oxigênio dissolvido com oxímetro YSI modelo 55, e o pH com peagâmetro digital YSI modelo 63.

\section{COLETA DO MATERIAL BIOLÓGICO}

Os peixes foram anestesiados (benzocaína, $50 \mathrm{mg} \mathrm{L}^{-1}$ ) e o sangue heparinizado retirado por punção caudal. Uma alíquota foi utilizada para determinação da glicose (King \& Garner, 1947), hemoglobina, hematócrito e número total de eritrócitos (aparelho Celm 550) e outra foi centrifugada para obtenção de plasma e análise de cortisol (Radioimunoensaio, Kit Coat-a-Count, DPC), cloreto (kit Labtest) sódio e potássio (seletor de íons Iselab Drake) e osmolaridade (Osmômetro Wescor Modelo 505).

\section{ANÁLISE ESTATÍSTICA}

O delineamento experimental foi em blocos casualizados, em esquema fatorial $3 \times 3+1$ ( 3 tempos de perseguição, 3 tempos após perseguição e 1 testemunha - sem manejo). Cada tratamento teve 4 repetiçóes (caixas), amostradas em dias diferentes (blocos) com 8 peixes em cada caixa.Os dados foram submetidos à análise de variância e as médias comparadas pelo teste de Tukey (5\%), pelo programa estatístico SAS (8.12). Os resultados estão apresentados com médias \pm erro padrão da média. 


\section{RESULTADOS E DISCUSSÃO}

O manejo de captura pode ser considerado como um agente estressor para algumas espécies de peixes e o efeito isolado da natação forçada imposta pela perseguição dos peixes com puçá pode ser verificado através de indicadores biológicos clássicos de estresse, como níveis sanguíneos de cortisol, glicose, cloreto, sódio, potássio, osmolaridade, hematócrito, número total de células vermelhas e da hemoglobina (Barton \& Iwama, 1991; Mazeaud \& Mazeaud, 1981; Morgan \& Iwama, 1997; Wendeelar Bonga, 1997).

O perfil das respostas fisiológicas após o exercício físico dos juvenis de matrinxã não apresentou as alterações típicas do estresse (Barton \& Iwama, 1991; Wendeelar Bonga, 1997). Até 60 minutos após o estímulo, não foram registradas alterações do cortisol plasmático $(P>0,05)$ nos peixes submetidos aos diferentes tempos de perseguição (Figura 1A). Do mesmo modo, a glicemia não apresentou mudanças associadas ao estímulo aplicado ( $\mathrm{P}>0,05$, Figura 1B). $\mathrm{O}$ aumento do cortisol significância estatística devido a grandes variaçōes individuais. As concentrações dos íons potássio não se alteraram após o exercício físico ( $\mathrm{P}>0,05$, Figuras 3C). As respostas iônicas reforçam os indicadores hormonais e metabólicos (cortisol e glicose sanguíneos), pois é sabido que os peixes regulam a concentração iônica interna dentre de limites estreitos e que mudanças no balanço eletrolítico sanguíneo ocorrem durante a resposta de estresse (McDonald \& Milligan, 1997). Durante o experimento, registrou-se, em todas as caixas, a temperatura $\left(25,7 \pm 0,7^{\circ} \mathrm{C}\right)$ e o nível de oxigênio dissolvido $(8,4 \pm 1,1 \mathrm{mg}$ $\left.\mathrm{L}^{-1}\right)$ e o $\mathrm{pH}(7,2 \pm 0,16)$ estando todos dentro das condiçōes ótimas de acordo com Boyd, 1984.

Respostas hematológicas também são usadas como indicadores de estresse em peixes (Morgan \& Iwama, 1997). Alterações no hematócrito, número de eritrócitos e concentração de hemoglobina indicam hemoconcentração ou hemodiluição causados por distúrbios osmorregulatórios (Houston, 1996). Entretanto, a natação forçada dos peixes não
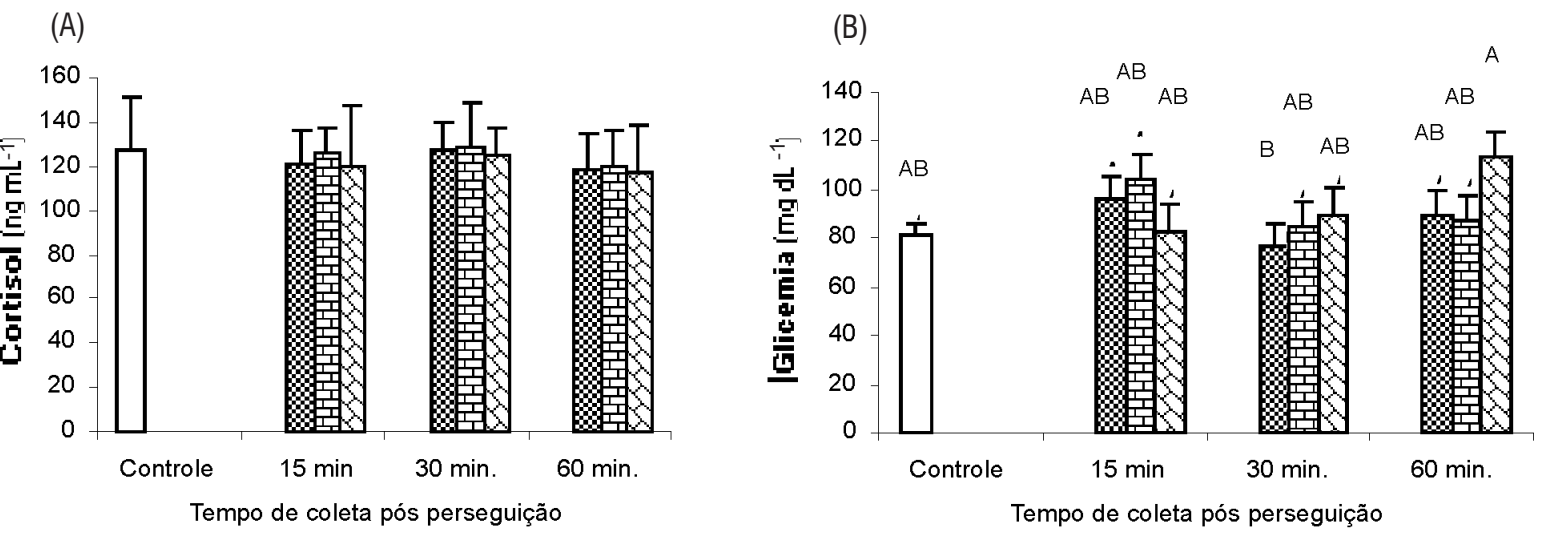

Figura 1 - Cortisol sangüíneo (A) e glicemia (B) de matrinxã aos 15, 30 e 60 minutos após perseguição de 2 《 ,5 田 e 10 区 minutos. Letras diferentes indicam diferenças $(P<0,05)$ entre os tempos após perseguição .

e da glicose no sangue são indicadores muito utilizados para expressar condição de estresse (Morgan \& Iwama, 1997; Wendelaar Bonga, 1997; Wells \& Pankhurst, 1999), o que sugere que o estímulo da perseguição aplicado no matrinxã não foi suficiente para gerar resposta de estresse suficiente para alterar esses indicadores.

Os níveis de cloreto plasmático foram reduzidos 15 minutos após a natação forçada $(\mathrm{P}<0,05$, Figura $3 \mathrm{~A})$, enquanto os níveis do sódio mais baixos foram registrados 60 minutos depois $(\mathrm{P}<0,05$, Figura $3 \mathrm{~B})$. Houve redução da osmolaridade a partir dos 30 minutos após o estímulo imposto aos peixes $(\mathrm{P}<0,01$, Figura 3D). Essas diferenças ocorreram da mesma forma independente do tempo de perseguição. Observou-se um comportamento de redução que, entretanto não teve interferiu nos valores de hematócrito (Figura 2B), número de eritrócitos (Figura 2A) e concentração de hemoglobina (Figura 2C) $(\mathrm{P}>0,05)$, corroborando com os outros indicadores, como a glicose e o cortisol.

A captura é uma prática muito comum em aqüicultura, como em caso de transferência de peixes de viveiros, transporte para outras instalações, biometria, seleção e manejo da reprodução artificial. Estudos prévios relatam que a captura que precede o transporte de peixes é responsável por gerar estresse mais intenso que o próprio transporte (Staurnes et al., 1994) e se caracteriza pelo caráter agudo e severo (Grutter \& Pankhurst, 2000). Entretanto, a captura compreende uma seqüência de estímulos que inclui a fase de perseguição e natação forçada do animal, a exposição do peixe ao ar e a 

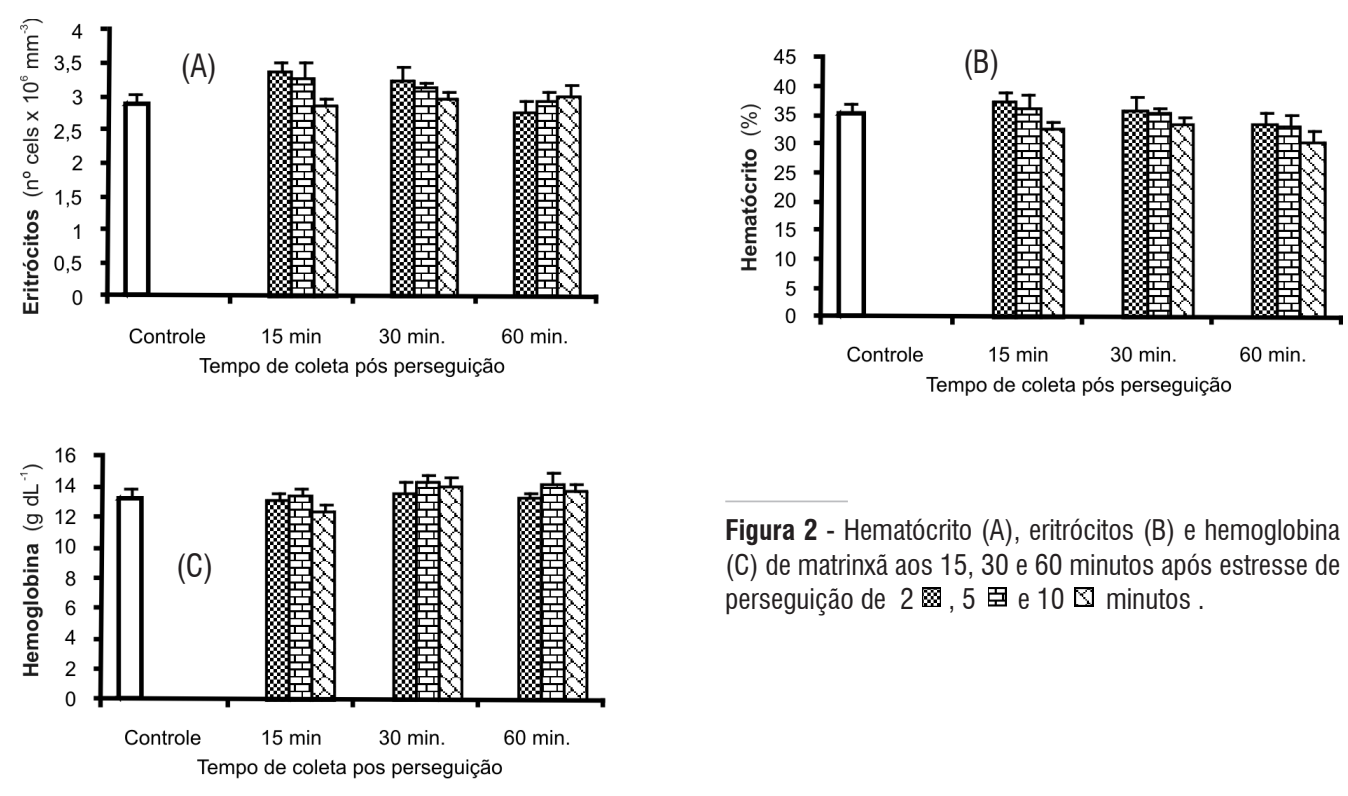

Figura 2 - Hematócrito (A), eritrócitos (B) e hemoglobina (C) de matrinxã aos 15, 30 e 60 minutos após estresse de

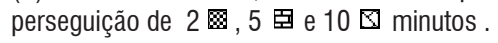

(A)

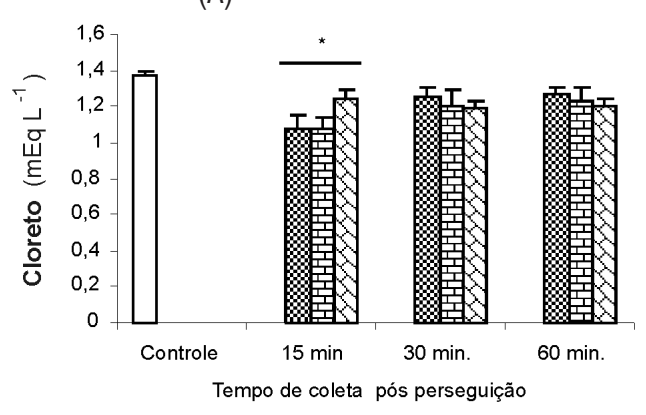

(C)

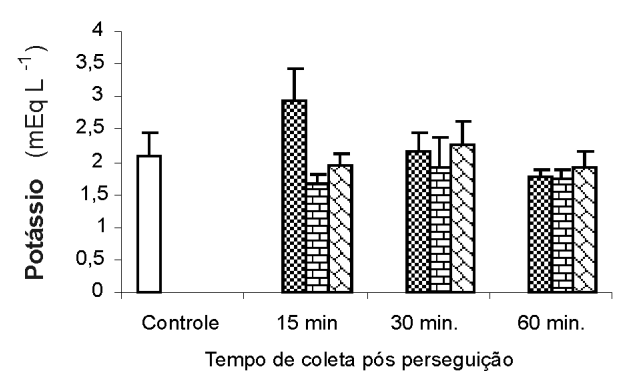

(B)
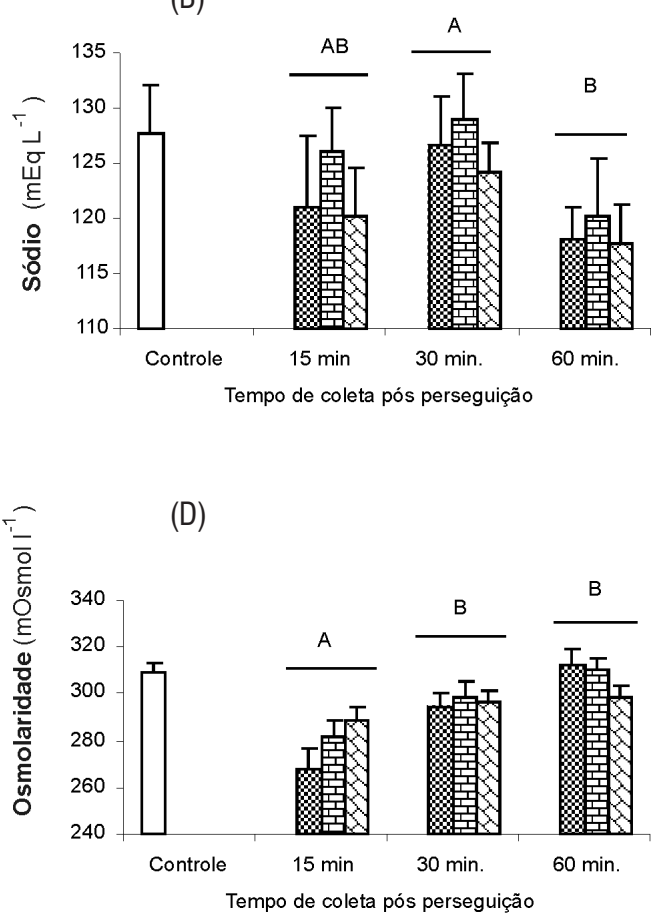

Figura 3 - Cloreto (A), sódio (B), potássio (C) e osmolaridade (D) sangüíneos de matrinxã aos 15, 30 e 60 minutos após perseguição de $2 \mathbb{Q}, 5$ 臣 e 10 minutos. Letras diferentes indicam diferenças $(P<0,05)$ entre os tempos após perseguição. 
abrasão do seu corpo com a rede e com outros peixes. Tanto o exercício físico da fuga (Milligan et al., 2000), como a hipóxia provocada pela retirada do peixe da água (Arends et al., 1999) e as injúrias na superfície do corpo (Ross \& Ross 1999) são estressores isolados e sucessivos que geram uma resposta de estresse acumulativa (Schreck, 2000). Entretanto, não se conhece a severidade e como o peixe se comporta em relação aos estressores individuais de uma sucessão deles, como no caso da captura. Os efeitos finais dos estressores na resposta fisiológica de estresse e no ajuste do organismo são dependentes da duração e severidade dos estressores individuais, da freqüência de situaçōes estressoras e do número e duração temporal entre as exposições (Schreck, 2000).

Evidências indicam que o estímulo para a liberação do cortisol não é o exercício físico em si, mas a inatividade pósexercício que promove períodos de recuperação mais longos (Wang et al., 1994) através das açóes de uma resposta de estresse mediada por cortisol. Na natureza, uma recuperação lenta não é conveniente, pois deixaria os peixes vulneráveis à predação, já que sua capacidade de atividade rápida para fuga estaria limitada. Foi observado que trutas selvagens, quando capturadas e em seguida liberadas, procuravam refúgio na água corrente e não em água parada (Milligan et al., 2000). Peixes em natação têm níveis de cortisol mais baixos que peixes em água parada (Boesgaard et al., 1993), sugerindo que a natação impõe menos estresse que a imobilidade. Estudo com truta, em condiçôes de laboratório, mostrou que peixes submetidos à 5 minutos de perseguição no tanque, se posteriormente colocados em água corrente de baixa velocidade, tinham os níveis de cortisol inalterados por período de tempo de 6 horas pós-exercício, enquanto que nos peixes deixados em água parada após a perseguiçãa, a concentração do cortisol aumentava uma hora depois do exercício (Milligan et al., 2000). Segundo os autores, os baixos níveis de cortisol induzem o uso do lactato produzido no músculo durante o exercício para síntese de glicogênio e, alternativamente, ocorre maior utilização de lactato em processos oxidativos (Milligan \& Farrel, 1991), para fornecer energia aeróbica ao peixe que continua nadando. Os músculos cardíaco, opercular e axial vermelho são os prováveis candidatos a uma aumentada utilização de lactato.

Tipicamente, no exercício físico, a elevação do cortisol plasmático é atrasada. Em geral, aumentos do cortisol plasmático não são observados até 30-60 minutos após o término do exercício (Milligan \& Wood, 1987) e os picos de liberação até 1-2 horas depois (Gamperl et al., 1994). Isto poderia explicar os resultados encontrados na resposta do cortisol do matrinxã, entretanto a ausência de alterações nos outros indicadores de estresse após exercício físico sugere ausência de estresse, pois a liberação de catecolaminas (adrenalina e noradrenalina) ocorre imediatamente após o exercício estressante (Milligan et al., 2000). Nesse caso, haveria aumento dos níveis de glicemia no matrinxã por ação glicogenolítica (Mazeaud \& Mazeaud, 1981), que não ocorreu, e, do mesmo modo, alteraçôes no balanço iônico. Também por ação adrenérgica, ocorre dilatação de vasos sanguíneos branquiais, por aumento da pressão sangüínea, para aumentar a perfusão nas lamelas e a captação de oxigênio a ser distribuído aos tecidos corporais (Wendelaar Bonga, 1997). O aumento da perfusão nas lamelas aumenta a permeabilidade do epitélio branquial e a perda de íons sódio e cloreto do sangue para o meio externo, no caso de peixes de água doce.

Em cinco espécies de ciprinídeos, a liberação de catecolaminas estimulou a contração esplênica e liberação de eritrócitos na corrente sanguínea de 3 a 30 minutos após início de exercício forçado, com redução de $63-85 \%$ do tamanho do baço e de $72-93 \%$ do conteúdo de hemoglobina (Yamamoto, 1987), enquanto que, em truta, exercício induzido por perseguição de 5 minutos promoveu contração do baço e aumento do hematócrito (Mendiola et al., 1997). Entretanto, nenhuma alteração nos parâmetros hematológicos foi observada no matrinxãa após a natação forçada por até 10 minutos.

A ausência de alteração nos principais indicadores de estresse como (cortisol plasmático, glicemia e parâmetros hematológicos) indica que a perseguição dos peixes com puçá e a natação forçada não foram estímulos intensos o bastante para o matrinxã e que o estresse que a espécie apresenta no manejo da captura que precede o transporte (Urbinati et al., 2004) é provocado pela ação acumulativa de todas as fases que compõem a captura. Estudo anterior com matrinxã mostrou que exemplares jovens colocados em contato com substância de alarme de extrato de pele de co-específicos apresentaram aumento da atividade motora no aquário sem apresentar alteração nos níveis sanguíneos de cortisol e glicose e de glicogênio hepático registrados em condiçôes de repouso (Ide et al., 2003). Adicionalmente, Rocha et al. (2004) não verificaram respostas claras de estresse em matrinxã mantido em alta densidade por 24 horas, reforçando os achados do presente trabalho no qual o matrinxã se mostrou bastante resistente ao manejo de captura.

\section{CONCLUSÃO}

O exercício intenso dos peixes por até 10 minutos não foi estímulo suficiente para gerar as principais respostas clássicas de estresse, ocorrendo somente leves alteraçōes em alguns parâmetros eletrolíticos, sugerindo que o matrinxã é resistente ao manejo de captura, prática comum na criação de peixes. 


\section{AGRADECIMENTOS}

Agradecimentos ao Conselho Nacional de Desenvolvimento Científico e Tecnológico (CNPq) pelo apoio financeiro, ao Centro de Aqüicultura da Unesp - Jaboticabal pela doação dos peixes, a sra. Damares Perecim Roviero pelo auxílio técnico e a todos do Laboratório de Peixes do Departamento de Morfologia e Fisiologia Animal, pelo apoio durante a pesquisa.

\section{BIBLIOGRAFIA CITADA}

Arends, R.J.; Mancera, J.M.; Muñoz, J.L.; Wendelaar Bonga, S.E.; Flik, G. 1999. The stress response of the gilthead seabream (Sparus aurata) to air exposure and confinement. Journal of Endocrinology, 163: 149-157.

Barton, B.A.; Iwama, G.K. 1991. Physiological changes in fish from stress in aquaculture with emphasis on the response and effects of corticosteroids. Reviews of Fish Diseases, 1: 3-26.

Boesgaard, L.; Nielsen, M.E.; Rosenkilde, P. 1993. Moderate exercise decreases plasma cortisol levels in Atlantic salmon (Salmo salar). Comparative Biochemistry Physiology, 106A: 641-643.

Boyd, C. E. 1984. Water quality in warmwater fishponds. 3ed. Auburn University. 334p.

Carneiro, P.C.F.; Urbinati, E.C. 2001a. Salt as a stress response mitigator of matrinxã Brycon cephalus (Teleostei: Characidae) during transport. Aquaculture Research, 32: 1-8.

Carneiro, P.C.F.; Urbinati, E.C. 2001b. Electrolyte disturbance in matrinxã Brycon cephalus following transport stress under benzocaine effect. Journal of Applied Aquaculture, 11: 1-13.

Carneiro P.C.F.; Urbinati, E.C. 2002. Transport stress in matrinxã, Brycon cephalus (Teleostei: Characidae), at different densities. Aquaculture International, 34: 1-9.

Clearwater, S.J.; Pankhurst, N.W. 1997.The response to capture and confinement stress of plasma cortisol, plasma sex steroids and vitellogenic oocytes in the marine teleost, red gurnard. Journal of Fish Biology, 50: 429-441.

Gamperl, A.K.; Vijayan, M.M.; Boutilier, R.G. 1994. Experimental control of stress hormone levels in fishes: techniques and applications. Rev. Fish Biol. Fish., 4: 215-225.

Gomes, L.C.; Roubach, R.; Cavero, B.A.S.; Pereira-filho, M.; Urbinati E. C. 2003 a. Transport of pirarucu Arapaima gigas in a closed system. Acta Amazônica, 33(4): 637-642.

Gomes, L.C.; Chippari-gomes, A. R.; Lopes, N.P.; Roubach, R.; Araujo-lima, C.A.R.M. E Urbinati, E.C. 2003b. Effect of fish density on the stress physiological responses and mortality of juvenile tambaqui Colossoma macropomum during transportation. Journal of the World Aquaculture Society, 34 (1): 76-84.

Grutter A.S.; Pankhurst, N.W. 2000. The effects of capture, handling, confinement and ectoparasite load on plasma levels of cortisol, glucose and lactate in the coral reef fish Hemigymnus melapterus. Journal of Fish Biology, 57: 391-401.

Houston, A.H.; Dobric, N.; Kahurananga, R. 1996. The nature of hematological response in fish. Fish Physiology and Biochemistry, 15: 339-347.
Ide, L.M.; Urbinati, E.C.; Hoffmann, A. 2003. The role of olfaction in the behavioral and physiological responses to conspecific skin extract in a teleost fish, Brycon cephalus. Journal of Fish Biology, 63: 332-343.

King, E.J.; Garner, R.J. 1947. Colorimetric determination of glucose. Journal of Clinical Pathology., 1: 30-33.

Mazeaud, M.M.; Mazeaud, F.; Donaldson, E.M. 1977.Primary and secondary effects of stress in fish: some new data with a general review. Transactions of the American Fisheries Society, 106: 201-212.

Mazeaud, M.M.; Mazeaud, F. 1981.Adrenergic responses to stress in fish. In Pickering, A.D.. Stress and fish. Academic Press, London. p. 49-75.

McDonald, G.; Milligan, C.L. 1997. Ionic, osmotic and acid-base regulation in stress. In Iwama, G.W.; Pickering, A.D.; Sumpter, J.P.; Schreck, C.B. (Eds.). Fish stress and health in aquaculture. Cambridge: University Press, p. 119-144.

Mendiola P.; Hernandez M.D.; De Costa J.; Zamora S. 1997. Trout plasma metabolites, blood factors and spleen contraction: responses to exercise. Revista Española de Fisiologia, 53(2): 217-23.

Milligan, C.L.; Wood, C.M. 1987. Regulation of blood oxygen transport and red cell $\mathrm{pHi}$ after exhaustive activity in rainbow trout (Salmo gairdneri) and starry flounder (Platichthys stellatus). Journal of the Experimental Biology, 133: 263-282.

Milligan, C.L.; Farrell, A.P. 1991. Lactate utilization by an in situ perfused trout heart: effects of workload and blockers of lactate transport. Journal of the Experimental Biology, 155: 357-373.

Milligan, C.L.; Hooke, G.B.; Johnson, C. 2000. Sustained swimming at low velocity following a bout of exhaustive exercises enhances metabolic recovery in rainbow trout. Journal of the Experimental Biology, 203: 921-926.

Morgan, J.D.; Iwama, G.K.; 1997. Measurements of stressed states in the field. In Iwama, G.W.; Pickering, A.D.; Sumpter, J.P.; Schreck, C.B. (Eds.). Fish stress and health in aquaculture. Cambridge: University Press, p. 247-270.

Pickering, A.D.; Pottinger, T.G. 1987. Poor water quality suppresses the cortisol response of salmonid fish to handling and confinement. Journal of Veterinary Biology, 30: 363-374.

Robertson, L.; Thomas, P.; Arnold, C.R.; Trant, J.M. 1987. Plasma cortisol and secondary stress responses of red drum to handling, transport, rearing density, and a disease outbreak. Progressive Fish-Culturist, 49: 1-12.

Rocha, R. M.; Carvalho, E.G.; Urbinati, E.C. 2004. Physiological responses associated with capture and crowding stress in matrinxã, Brycon cephalus, (Gunther, 1869), Teleostei: Characidae. Aquaculture Resarch, 35: 1-5.

Ross, L.G.; Ross, B. 1999. Anesthetic and sedative techniques for aquatic animals. Blackwell Science, Oxford, UK.

Schreck, C.B. 2000. Accumulation and long-term effects os stress in fishes. In: Moberg G.P.; Manch, J.A. (Eds.) The Biology of Animal Stress. CAB International, p. 147-158.

Selye, H. 1950. Stress and the general adaptation syndrome. British Medical Journal, 1: 1383-1392. 


\section{ACTA}

Staurnes, M.; Sigholt, T.; Pedersen, H.P.; Rustad, T. 1994. Physiological effects of simulated high-density transport of Atlantic cod (Gadus morhua). Aquaculture, 119: 381-391.

Urbinati, E.C.; Carneiro, P.C.F. 2001. Metabolic and hormonal responses of the matrinxã Brycon cephalus (Teleostei: Characidae) to the stress of transport under the influence of benzocaine. Journal of the Aquaculture in the Tropics, 16(1): 75-85.

Urbinati, E.C.; Carneiro, P.C.F. 2004. Práticas de Manejo e Estresse dos Peixes em Piscicultura Intensiva. In Cyrino, J.E.P.; Urbinati, E.C.; Castagnolli, N. (Eds.). Tópicos Especiais em Piscicultura Tropical. Editora TecArt. p. 171-193.

Urbinati, E.C.; Abreu, J.S.; Camargo, A.C.S.; Landines, M.A. 2004. Loading and transport stress in juvenile matrinxã (Brycon cephalus) at various densities. Aquaculture, 229: 389-400.
Wang, Y.; Heigenhauser, G.J.F.; Wood, C.M. 1994. Integrated responses to exhaustive exercise and recovery in rainbow trout white muscle: acid-base, phosphogen, carbohydrate, lipid, ammonia, fluid volume and electrolyte metabolism. Journal of the Experimental Biology, 195: 227-258.

Wells, R.M.G.; Pankhurst, N.W. 1999. Evaluation of simple instruments for the measurement of blood glucose and lactate, and plasma protein a stress indicator in fish. Journal of the World Aquaculture Society, 30: 276-284.

Wendelaar Bonga, S.E. 1997. The stress response in fish. Physiological Reviews,77: 591-625.

Yamamoto K. 1987. Contraction of spleen in exercised cyprinid. Comparative Biochemistry and Physiology,,87A: 1083-1087.

Recebido em 10/01/2008

Aceito em 06/02/2009 
